\title{
AERODYNAMICAL INVESTIGATION OF THE WINGS OF AN ULTRALIGHT AIRCRAFT
}

\author{
Richárd MOLNÁR ${ }^{1}$, Gergely DEZSŐ ${ }^{2}$ \\ University of Nyíregyháza, Institute of Engineering and Agricultural Sciences, Department of Transportation \\ Engineering and Infitechnology, Nyíregyháza, Hungary \\ ${ }^{1}$ molnarrichard@freemail.hu \\ ${ }^{2}$ dezso.gergely@nye.hu
}

\begin{abstract}
Nowadays more and more ultralight aircraft are being built because the building process itself and the acquisition of the necessary documentation is relatively easy. Furthermore, these planes are easier to fly than larger types of aircraft. This article presents the engineering work and documentation that is necessary for the building process. The calculations can be done traditionally on paper which is an extremely complex task. With the innovations and developments in the technical field though, it is possible now to simplify these calculations, the basis of which is the finite element method and aerodynamics simulations. If the finite element method is adequate, the boundary conditions are ideal and input-output settings for the simulations are correct, it is possible to compare the traditional calculations to the modern simulated engineering work, thus the time necessary for achieving precise results becomes significantly shorter.
\end{abstract}

Keywords: ultralight aircraft, finite element method, aerodynamics simulations.

\section{Planning procedure}

\subsection{Traditional planning procedure}

The entire process of engineering is possible by traditional calculations if we consider the mandatory prescribed rules, regulations and basic requirements. The aim of the engineering work is to determine the solidity scaling, for which it is necessary to define the stress that comes into effect at any given cross-sections of the wing. Following that we can determine the location of the cross-section that will be exposed to the greatest amount of stress, and based on that we can decide upon the necessary material types and thicknesses. The traditional building procedure steps are as follows:

- determination of air speed;

- delimitating the load areas;

- calculating the distribution of load in the wing [2];

- determining the resultant of the distributed load of the wing.

If we integrate the distributed loads (which is a rather lengthy procedure that require great precision) we obtain values for the stress.

\subsection{Simulation planning procedure}

Due to modern simulation software, we can now locate the biggest stress in the cross-section faster and without the previous lengthy and complicated procedures. The essential part is the creation of the adequate final element method and the determination of the correct boundary conditions. Thereby our solution is rather exact, it reflects reality and therefore we can use it in the next phases of our calculations, (solidity scaling, and determination of materials)

\section{Model}

We simulated and analyzed the airflow fields around the wing with diverse parameter settings regarding travel speed and angle of attack. In order to do that, we had to make the necessary sensitivity measurements such as finite element method sensitivity examinations and turbulence model sensitivity examinations. We studied two models. (Figure 1. and Figure 2.) 


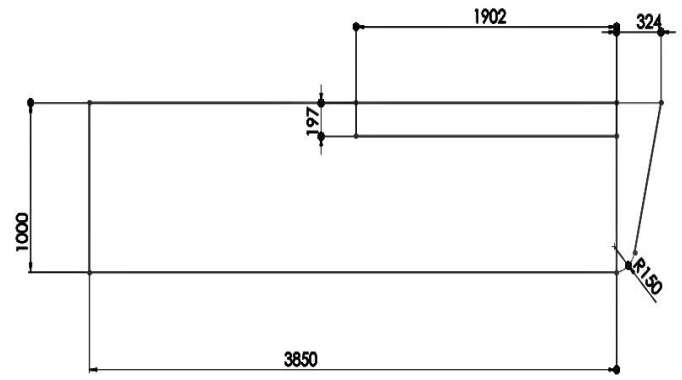

Figure 1. Top view of the wing

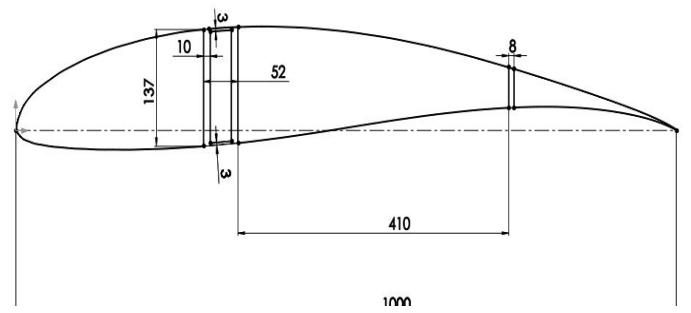

Figure 2. The cross section of the wing

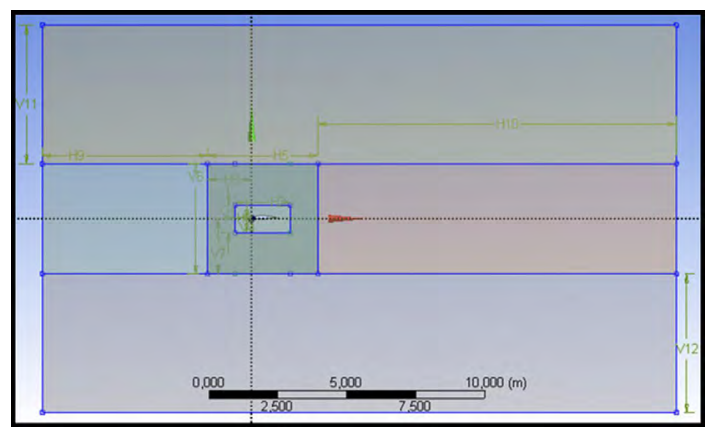

Figure 3. Endless wing model (We divided this model into six parts, because that way we were able to create the most detailed finite element method. This is very important, because regarding the calculations, the most essential part is the boundary layer)

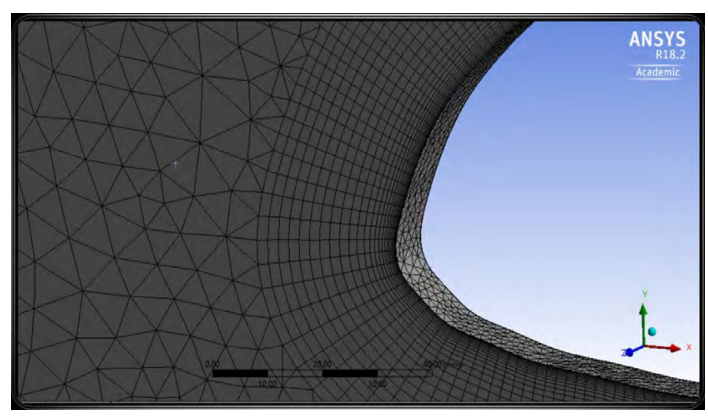

Figure 4. Finite element method sensitivity at the leading edge. (Our target was to obtain the smallest possible finite element measurements along the boundary layer.)

\subsection{Endless wing model}

This model is standard in rigid wing hydrodynamics examinations. The essential characteristic of this is that we presume the existence of a displacement symmetry in the airflow around the wing. That means that it is sufficient to know the air flow data of just one narrow portion of the wing's cross-section, by displacement we can extrapolate these results for the other parts too. Therefore this model does not in fact examine an endless wing, it only examines a small slice of the wing. This kind of model is suitable for determining the pressure and velocity dispersion around the wing, and also the lift coefficient and drag coefficient. An important advantage of the endless wing model is that the examined area is not too big, therefore the time necessary for the calculations is shorter and the solutions are relatively precise, furthermore it does not require expensive hardware. However it also has a disadvantage: this model gives us no information or data regarding the vortex that begins at the tip of the wing and which is larger by orders of magnitude than the entire plane itself. But here we do not examine this phenomenon. In the first study we assumed an endless wing and we made our calculations considering a $100 \mathrm{~mm}$ long slice of such a wing, assuming adequate symmetry and boundary conditions. Another advantage of this method is that it allows for an extremely detailed finite element method. (Figure 3.)

On the other hand we cannot really study air circulation, which plays an important role in aerodynamics. We could determine a link between lift coefficient and drag coefficient. In more traditional engineering this information could only be obtained as data from external sources.

In order to obtain the most precise solution, we have to find the adequate settings, beginning with the finite element method. We have to reduce the size of the finite elements until it no longer influences the solution. This is referred to as final element method sensitivity examination. This reduction of the element size was done in the area around the wing. (Figure 4.)

\subsection{Finite wing model}

The aim of the finite wing model (a three dimension wing) is to facilitate examinations of diverse characteristics, the most important of which is pressure dispersion from the wing root to the wingtip. When we created the model we encountered a serious problem due to the fact that this 
model is not $100 \mathrm{~mm}$ but $12 \mathrm{~m}$ wide so that the number of elements suddenly increased from an order of magnitude of hundreds of thousands to one of millions. As such the finite element method is not detailed enough, therefore the obtained solutions are rather imprecise. The three dimension area's partition is similar to that of the endless wing model. We have to place the half wing that is the object or our research (the size of which is $5,185 \mathrm{~m}$ ) into a three dimension model that is big enough to contain the effects caused by such a finite wing (the wingtip vortex), therefore, we created a 12 m wide „box”. In this case the most important thing that we examined is the pressure dispersion through the wingspread and its numerical expressions. Once we obtained the specific data, we were able to compare them with those obtained by traditional calculations. (Figure 5.)

\section{Evaluation of data}

In the case of finite wing lift, dispersion becomes elliptical, because pressure is equalized at the wingtip, therefore at that spot $F_{\text {lift }}=0$. This phenomenon can be seen in Figure 6 . We created an $\mathrm{x}-\mathrm{y}$ plain, $0,5 \mathrm{~m}$ from the $\mathrm{x}$ axis (half of the length of the chordline), on this plane we marked the pressure dispersion. We can see that under the wing the pressure is higher than in the upper area. Towards the wing-tip, this pressure difference decreases until it equalizes.

In the next step, we created an $x-y$ plane. This plane can be placed anywhere throughout the wing spread allowing us to evaluate the value of pressure dispersion in any given slice. During the traditional calculation we examined 11 different spots on the half wing and we made these examinations in the ANSYS as well. It possible to obtain the maximum and minimum pressure values for the given cross-section. From this data we calculate pressure difference, then we multiply that with the lift coefficient (considering the angle of the attack) and with the area of the wing section. This solution is the basis of comparison (Pn). In the next phase we illustrated the Pn values obtained by traditional methods and those from the simulations for a given airspeed and angle of attack value. (Figure 7. „A” examined case).

From Figure 7. it is obvious that the two solutions are very similar. The difference of the wavy pattern is caused by the fact that due to technical reasons we could not create the adequate finite element method, the quality of finite element approximation for an aero-dynamic model of this

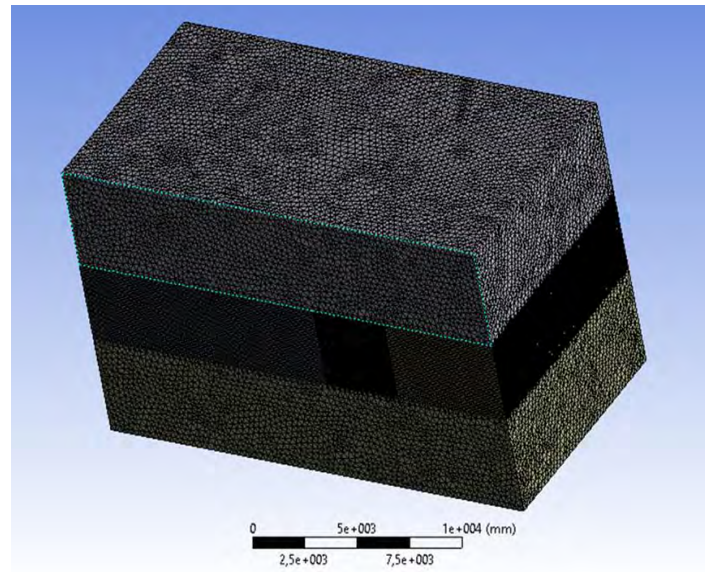

Figure 5. Finite wing model (Calculations times and system requirements were significantly increased with bigger size models.)

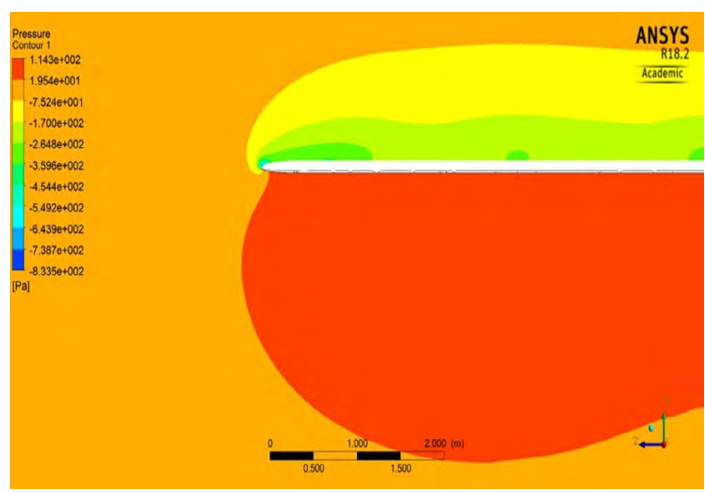

Figure 6. Pressure distribution in the solution of the finite wing model

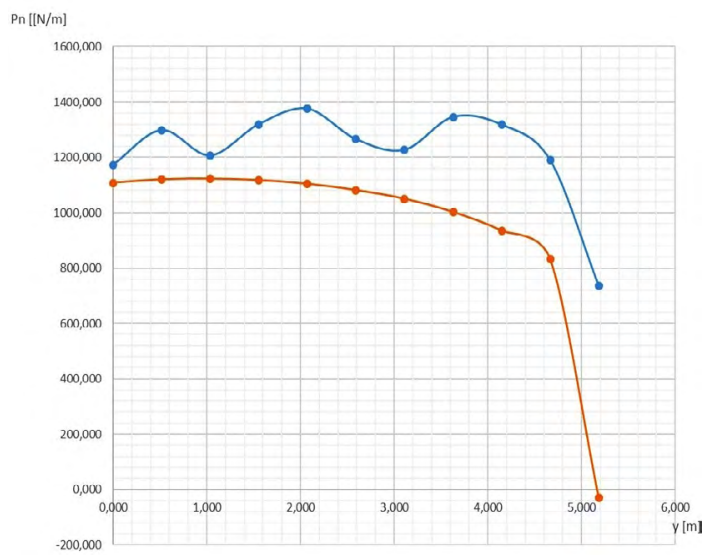

Figure 7. Comparison (The upper curve is the simulation solution, the lower one is the traditional solution.) 


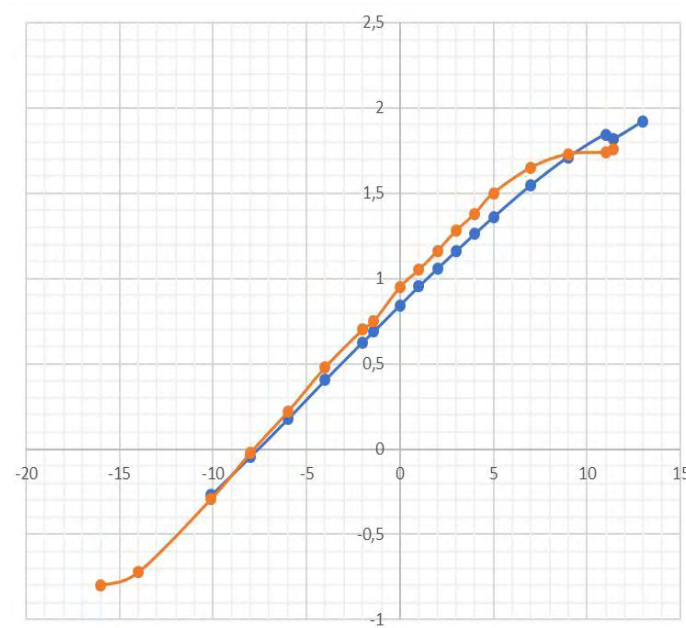

Figure 8. Comparison of lift coefficient (CL) values gained from classical calculations (blue) and simulations (orange) size can only be improved by increasing the number of finite elements, but this results in significantly higher computational cost.

In Figure 8. We show that lift coefficients calculated by simulation closely match values obtained from classical calulations.

\section{Conclusion}

We conclude that both simulation strategies are suitable for obtaining relevant data that can be used quantitatively. These results closely match those obtained by the usual means of paper-based calculations that have already been confirmed in numerous experiments.

\section{References}

[1] Petur L.:Repülőgép szilárdságtan. Tankönyvkiadó vállalat, Budapest, 1952.

[2] Rácz E.: Repülőgéptervezés. Tankönyvkiadó, Budapest, 1955. 